\title{
Unsteady MHD Flow of Nanofluid with Variable Properties over a Stretching Sheet in the Presence of Thermal Radiation and Chemical Reaction
}

\author{
Musa Antidius Mjankwi $\mathbb{D}^{1},{ }^{1}$ Verdiana Grace Masanja, ${ }^{1}$ \\ Eunice W. Mureithi, ${ }^{2}$ and Makungu Ng'oga James ${ }^{2}$ \\ ${ }^{1}$ Department of Mathematics, The Nelson Mandela African Institution of Science and Technology (NM-AIST), \\ P.O. BOX 447, Arusha, Tanzania \\ ${ }^{2}$ Department of Mathematics, University of Dar es Salaam (UDSM), P.O. BOX 35062, Dar es Salaam, Tanzania \\ Correspondence should be addressed to Musa Antidius Mjankwi; mjankwim@nm-aist.ac.tz
}

Received 28 December 2018; Revised 24 March 2019; Accepted 7 April 2019; Published 2 May 2019

Academic Editor: Hernando Quevedo

Copyright (C) 2019 Musa Antidius Mjankwi et al. This is an open access article distributed under the Creative Commons Attribution License, which permits unrestricted use, distribution, and reproduction in any medium, provided the original work is properly cited.

\begin{abstract}
The unsteady magnetohydrodynamics (MHD) flow of nanofluid with variable fluid properties over an inclined stretching sheet in the presence of thermal radiation and chemical reaction is studied taking into account the effect of variable fluid properties in thermal conductivity and diffusion coefficient. The governing partial differential equations are transformed into ordinary differential equations by using similarity transformation. The numerical solutions of the problem are obtained by using the fourth order Runge-Kutta method in line with the shooting technique. It is found that the increase in both thermal conductivity and radiative heat flux decreases the heat transfer rate but increases the skin friction and mass transfer rates. It is further observed that the increase in porosity parameter and magnetic field reduces the skin friction, heat, and mass transfer rates.
\end{abstract}

\section{Introduction}

The study concerned with the fluid flow over a stretching sheet has created many applications in industries among which are polymer industries, aerodynamic extrusion of plastic sheets, glass-fiber production, condensation process of a metallic plate in a cooling bath, and glass. Such studies were first initiated by Sakiadis [1] who simplified the convection term in the momentum equation by assuming that all convections take place at a constant velocity of a moving object. Following this pioneering work, the study of fluid flow over a continuous surface moving with either constant or variable velocity received wide attention among researchers such as Crane [2], Chen and Char [3].

Erickson et al. [4] extended the Sakiadis [1] study by introducing the effects of momentum, heat transfer, and mass transfer on the surface that is moving at constant speed. This study was further extended by Gupta [5] who added new parameters of suction, injection and considered the sheet to be stretched with a linear speed. In all of these studies, the commonly coolant fluids used were oils, water, and ethylene glycol; however, these fluids had relatively low thermal conductivity and stability.

In recent year, it has been proposed to mix up nanoparticles (10-50nm) and base fluids (oils, water, and ethylene glycol) to form the fluid commonly known as nanofluids. The presence of nanoparticles in these fluids has significant effects on the physical properties of the fluid and causes a major increase in the heat transfer as presented by Hussain et al. [6] and Hussain et al. [7]. The presence of magnetic nanoparticles in these fluids makes nanofluid to be among the electrically conducting fluids. The study of magnetic properties and the behavior of electrically conducting fluids are well known as hydromagnetics or magnetofluid dynamics or magnetohydrodynamics (MHD).

MHD laminar boundary layer flow behavior over a stretching surface is an important type of flow with considerable practical applications in electrochemistry, chemical engineering, geophysics, astrophysics, and polymer processing. This is due to the fact that MHD absorbs energy and produces 
a controllable behavior; as a result, it can be controlled and directed by using external magnetic fields and hence slowing the rate of solidification of the cooled material and improving its mechanical properties as presented by Makinde [8], Hussain et al. [9], and Hussain et al. [6].

The characteristic of nanofluid under the effect of magnetic fields becomes the most attractive method in this process as it is simple to use and because of its uniqueness and nature. Hussain [10] in his study on the effect of MHD flow of nanofluids with heat and mass transfer through a porous media with thermal radiation, viscous dissipation, and chemical reaction observed that the dimensionless velocity profiles of nanofluids decrease as the magnetic field and permeability of the porous medium parameter increase.

The same results were observed by Ahmmed et al. [11] while performing the research in unsteady MHD free convection flow of nanofluid through an exponentially accelerated inclined plate embedded in a porous medium with variable thermal conductivity in the presence of thermal radiation. Experimentally, this finding means as the strength of external magnet increases, the flow speed of the magnetic nanofluid decreases; hence, nanofluids cool the intended object such as sheets or plates.

Haile and Shankar [12] in their study on the effects of thermal radiation, viscous dissipation, and chemical reaction on heat and mass transfer of MHD flow of nanofluids through a porous medium observed that as the thermal radiation or viscous dissipation increases, it causes increase in temperature of the coolant fluid. Also, as the chemical reaction parameter increases, the concentration of fluid decreases in the boundary layer flow. Uddin et al. [13] while analyzing the free convection flow of magnetic nanofluid with chemical reaction observed that as the flow velocity is reduced by the magnetic field, the temperature of the fluid increases.

Mehmood et al. [14] while performing the research on the effects of nonlinear thermal radiation and inclined magnetic field based on MHD in aluminum water nanofluid observed that the increase in magnetic field inclined angle results in the decrease in average Nusselt number. Hussain et al. [15] studied the effects of inclined angle on mixed convection nanofluid flow in a double lid-driven with discrete heat source and concluded that the increase in inclination angle causes the increase in average Nusselt number.

Sheikholeslami et al. [16] in their study on the effects of magnetic field on the forced convection flow of nanofluids over a stretching surface concluded that momentum boundary layer thickness decreases as the magnetic parameter increases. However, in the research presented by Haile and Shankar [12], Uddin et al.[13] and Sheikholeslami et al. [16], the physical parameters of the coolant fluid such as fluids viscosity, thermal conductivity, diffusion coefficient, and fluid density were treated constantly. In fact, the temperature of the hot stretched sheet causes the temperature of the coolant fluid to change. This effect makes the physical parameters of the coolant fluid to be temperature dependent.

For this reason some researchers, for instance, James et al. $[17,18]$, in their studies considered the effects of temperaturedependent viscosity in the steady flow and observed that an increase in viscosity results in the increase of both heat and mass transfer rate. This means the fluid with higher viscosity cools well the hot stretched sheet. Reddy and Chamkha [19] observed that an increase in thermal conductivity increases the temperature of the coolant fluid. Also an increase in variable diffusion coefficient resulted in the increase of concentration of the coolant fluid [20]. However, the flow under consideration in all the above studies was steady flow of the fluid.

Based on the few researches that have been presented by James et al. [17, 18], Reddy and Chamkha [19], the variations of fluid properties of the coolant nanofluid should be considered when analyzing the heat and mass transfer flow over a hot sheet or plate because they play an important role during the cooling processes. Proper consideration of these effects will lead to the higher increase of the mechanical properties of the cooled materials such as the strength of the sheets or plates. However, all of the studies cited above were governed by the steady flow of which by nature it is not true.

In real situations, the flow of fluids induced by stretched sheets involving heat transfer is unsteady in nature due to the sudden motion of the stretched sheet, change of temperature of the sheet and that of fluids and also due to the sudden change of concentration of nanofluids. The study becomes significant when the flow is unsteady and with variable physical parameters as presented by Hunegnaw and Kishan [21], Shukla and Rana [22], Sulochana and Kumar [23], and Venkataramanaiah [24].

Experimental studies have established that the physical properties of magnetic nanofluids change with varying average particles size, changing in nanoparticle shape and the nature of base fluid used as presented by Rao and Ranganayakulu [25]. These findings influenced researchers like Mutuku [26] who proposed the modifications of some cooling systems (e.g., car radiators) or cooled materials (e.g., stretched sheet) by including the effect of external magnetic field against nanofluid (coolant fluids) so as to control the fluid flow for effective cooling.

If the flow speed of magnetic nanofluid will be controlled or directed by external magnetic fields, the magnetic nanofluid will stay at the same hot point for some time. This will cause the fluid properties such as density, thermal conductivity, diffusion coefficient, and fluid viscosity to vary with respect to temperature. Consequently, for higher variations it might cause the effects in cooling processes and, thus, further studies on the effect of variation of fluid properties with respect to temperature are needed.

In most cases, the thermal conductivity is modeled as a linear function of temperature. On the other hand, the diffusion coefficient (D) depends on molecular size, temperature, pressure, and other properties of the diffusing substance. This means, with an exception of temperature, if all other parameters are kept constant, then the diffusion coefficient can be modeled as a function of temperature. Alsabery et al. [27] in their experimental research on conjugate natural convection of $\mathrm{Al}_{2} \mathrm{O}_{3}$-water nanofluid in a square cavity with a concentric solid modeled the diffusion coefficient as linear function of temperature based on Einstein-Stoke's equation for Brownian diffusion coefficient. 
Gharagozloo and Goodson [28] in their experimental research based on temperature-dependent aggregation and diffusion in nanofluids modeled the diffusion coefficient to depend on temperature. In this study they observed that as the diffusion is slowed, it causes the reduction in concentration distribution. In view of several analogies between heat and mass transfer during cooling process, for instance in solidification of binary alloy and in extrusion of sheet based on coolant fluids such as nanofluids, one can model both thermal conductivity and diffusion coefficient as a liner function of temperature.

Inspired and motivated by the current research, the dependency of thermal conductivity and mass diffusivity on temperature occurs in many natural phenomena (e.g., photosynthesis) and technological processes like drying crystals, cooling of nuclear reactors, solar ponds, cooling process during solidification of binary alloy, and extrusion of sheet. Thus, the behavior of concentration and temperature of nanofluid under the effects of variation of thermal conductivity and diffusion coefficient as a function of temperature in the presence of thermal radiation and chemical reaction when nanofluids are used as coolant fluids needs investigations.

However, to the authors' knowledge there are no studies that have been reported on unsteady MHD flow of nanofluids with variable fluid properties on both thermal conductivity and diffusion coefficient with regard to temperature change, over an inclined and permeable stretching sheet in the presence of thermal radiation and chemical reaction. The problem has an important application on cooling process for the purpose of improving mechanical properties of heated sheet that cannot be cooled by the use of traditional methods based on the base fluids.

In this research the model equations will be transformed into ordinary differential equations by using similarity transformation. Numerical shooting technique together with the fourth order Runge-Kutta scheme will be used to obtain numerical solutions of the model. The effects of nondimensional governing parameters such as variable thermal conductivity, variable diffusion coefficient, radiative heat flux, chemical reaction, porous medium, unsteadiness parameter, magnetic parameter, Prandtl number, Eckert number, Schmidt number, concentration Grashof number, thermal Grashof number, inclination angle and suction velocity parameter on dimensionless velocity, temperature, concentration profiles as well as skin friction, and Nusselt and Sherwood numbers will be discussed when magnetic nanofluids are applied as coolant fluid under the effects of external magnetic fields.

\section{Formulation of the Problem}

Consider unsteady two-dimensional incompressible laminar boundary layer MHD flow of a viscous nanofluid over a permeable inclined stretching sheet. The nanofluid is supplied heat by the stretching sheet and concentration of chemical species at uniform rates. It is assumed that the influence of density variation with temperature and concentration occurs only on the body force term and hence the changes in both

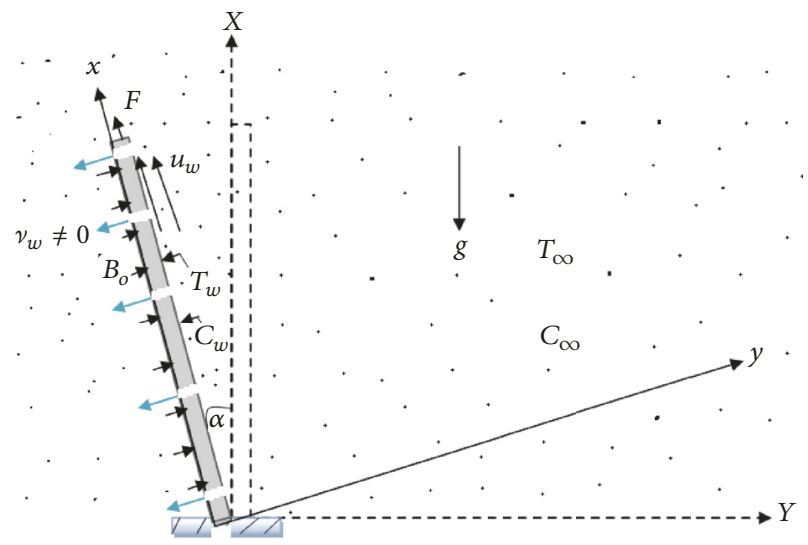

FIGURE 1: Inclined flow near the sheet.

concentration and temperature induce the buoyancy force. A uniform magnetic field is applied normal to the surface of the stretching sheet. Further, it is assumed that a homogeneous first order chemical reaction with thermal radiation is taking place in the flow. It is assumed that the velocity of the stretching sheet is $u_{w}(x, t)$ in the direction of the force $F$ applied along the $x$-axis and that of the mass transfer is $v_{w}(t)$ normal to the stretched sheet. It is also assumed that the surface (wall) temperature and concentration of the sheet are $T_{w}(x, t)$ and $C_{w}(x, t)$, respectively, while the uniform temperature and concentration far from the sheet are, respectively, $T_{\infty}$ and $C_{\infty}$. In addition, it is assumed that the effect described by Fourier's and Fick's law is of higher order of magnitude than the effect due to Dufour and Soret and thus the Dufour and Soret effects are neglected. The fluid thermal conductivity and molecular diffusivity are assumed to vary as a linear function of temperature. The model flow diagram is illustrated in Figure 1.

Under these assumptions with the Boussinesq and boundary layer approximations, the governing model equations are given by equations (1) to (4):

$$
\begin{aligned}
\frac{\partial u}{\partial x}+\frac{\partial v}{\partial y}= & 0 \\
\frac{\partial u}{\partial t}+u \frac{\partial u}{\partial x}+v \frac{\partial u}{\partial y}= & v^{*} \frac{\partial^{2} u}{\partial y^{2}}-\frac{v^{*}}{\kappa^{*}} u-\frac{\sigma B_{0}^{2}}{\rho} u \\
& +g \beta_{T}\left(T-T_{\infty}\right) \cos \alpha \\
& +g \beta_{C}\left(C-C_{\infty}\right) \cos \alpha \\
\frac{\partial T}{\partial t}+u \frac{\partial T}{\partial x}+v \frac{\partial T}{\partial y}= & \frac{1}{\rho C_{p}} \frac{\partial}{\partial y}\left(K(T) \frac{\partial T}{\partial y}\right)-\frac{1}{\rho C_{p}} \frac{\partial q_{r}}{\partial y} \\
& +\frac{\mu}{\rho C_{p}}\left(\frac{\partial u}{\partial y}\right)^{2} \\
\frac{\partial C}{\partial t}+u \frac{\partial C}{\partial x}+v \frac{\partial C}{\partial y}= & \frac{\partial}{\partial y}\left(D(T) \frac{\partial C}{\partial y}\right)-K_{c}\left(C-C_{\infty}\right)
\end{aligned}
$$


where $t$ is time, $u$ and $v$ are the velocity components in the $x$ and $y$ direction, respectively, $\rho$ is the mass density of the fluid, $\mu$ is the coefficient of viscosity, $C_{p}$ is the specific heat capacity at constant pressure, $\kappa^{*}$ is the permeability, $K$ is the thermal conductivity, $D$ is the molecular diffusivity, and $K_{c}$ is the rate of chemical reaction. $\beta_{T}$ is the coefficient of volumetric thermal expansion, $\beta_{C}$ is the coefficient of volumetric expansion due to chemical species, $T$ is the temperature of the species, $C$ is the concentration of the species, $T_{\infty}$ and $C_{\infty}$ are ambient temperature and concentration, respectively, $v^{*}$ is the kinematic viscosity of the ambient fluid, $\sigma$ is the electrical conductivity, $B_{0}$ is the strength of external magnetic field, $g$ is the acceleration due to gravity, $q_{r}$ is the radiation heat flux, and $\alpha$ is the angle of inclination measured from the vertical axis to the stretching sheet.

The relevant boundary conditions are given in (5):

$$
\begin{array}{ll}
u & =u_{w}(x, t)=\frac{c x}{1-\lambda t}, \\
v & =v_{w}(t), \\
T & =T_{w}(x, t), \\
C & =C_{w}(x, t) \\
u \longrightarrow 0, & \text { at } y=0 \\
T \longrightarrow T_{\infty}, & \\
C \longrightarrow C_{\infty} & \text { as } y \longrightarrow \infty
\end{array}
$$

where $c$ is the initial stretching rate, $\lambda$ is a constant, and the subscripts $w$ and $\infty$ stand for the sheet and boundary layer edge (ambient conditions), respectively. The wall mass transfer velocity (or the fluid suction velocity) then becomes $v_{w}=-v_{0} \sqrt{v^{*} c /(1-\lambda t)}$, and $v_{0}$ is a constant that describes the wall mass transfer parameter with the meaning that when $v_{0}>0$, we have a suction, when $v_{0}=0$, we have an impermeability, and when $v_{0}<0$, we have an injection. The radiation heat flux $\left(q_{r}\right)$ is modeled by using the Rosseland's (1972) approximation given in (6):

$$
q_{r}=-\left(\frac{4 \sigma^{*}}{3 k_{1}}\right) \frac{\partial T^{4}}{\partial y}
$$

where $\sigma^{*}$ is the Stefan- Boltzmann constant and $k_{1}$ is the coefficient of absorption. It is also assumed that if the difference in temperature within the flow is $T^{4}$, then $T^{4}$ can be expressed as a linear combination of the temperature by expanding the $T^{4}$ by Taylor's series about $T_{\infty}$ to obtain (7):

$$
T^{4}=T_{\infty}^{4}+4 T_{\infty}^{3}\left(T-T_{\infty}\right)+6 T_{\infty}^{2}\left(T-T_{\infty}\right)^{2}+\cdots
$$

If we neglect the higher order beyond the first degree in $\left(T-T_{\infty}\right)$ in this series and opening brackets on the right-hand sides of (7) we obtain (8):

$$
T^{4} \approx-3 T_{\infty}^{4}+4 T_{\infty}^{3} T
$$

Substituting the right-hand side of (8) into (6) for $T^{4}$ yield (9):

$$
\begin{aligned}
q_{r} & =-\left(\frac{4 \sigma^{*}}{3 k_{1}}\right) \frac{\partial T^{4}}{\partial y}=-\left(\frac{4 \sigma^{*}}{3 k_{1}}\right) \frac{\partial}{\partial y}\left(-3 T_{\infty}^{4}+4 T_{\infty}^{3} T\right) \\
& =-\left(\frac{16 T_{\infty}^{3} \sigma^{*}}{3 k_{1}}\right) \frac{\partial T}{\partial y}
\end{aligned}
$$

The rate of change in radiative heat flux with respect to $y$ as presented by Mukhopadhyay [29] is given by (10):

$$
\frac{\partial q_{r}}{\partial y}=-\left(\frac{16 T_{\infty}^{3} \sigma^{*}}{3 k_{1}}\right) \frac{\partial^{2} T}{\partial y^{2}}
$$

Substitution of (10) into the second term on the right-hand side of (3) changes (3) into (11):

$$
\begin{aligned}
\frac{\partial T}{\partial t}+u \frac{\partial T}{\partial x}+v \frac{\partial T}{\partial y}= & \frac{1}{\rho C_{p}} \frac{\partial}{\partial y}\left(K(T) \frac{\partial T}{\partial y}\right) \\
& +\frac{1}{\rho C_{p}}\left(\frac{16 T_{\infty}^{3} \sigma^{*}}{3 k_{1}}\right) \frac{\partial^{2} T}{\partial y^{2}} \\
& +\frac{\mu}{\rho C_{p}}\left(\frac{\partial u}{\partial y}\right)^{2}
\end{aligned}
$$

The temperature of the fluid at the surface of the sheet $T_{w}(x, t)$ and the concentration of the fluid at the surface of the sheet $C_{w}(x, t)$ are functions of time $t$ and distance $x$ and are assumed to be of the forms presented in (12).

$$
\begin{gathered}
T_{w}(x, t)=T_{\infty}+\frac{b x}{(1-\lambda t)^{2}} \\
C_{w}(x, t)=C_{\infty}+\frac{b x}{(1-\lambda t)^{2}}
\end{gathered}
$$

where in the absence of buoyancy force $b=0$, which corresponds to the limit of forced convection.

2.1. Similarity Transformation. The partial differential equations (2), (4), and (11) are transformed into ordinary differential equations by introducing the dimensionless variables given by (13):

$$
\begin{aligned}
\eta & =\sqrt{\frac{c}{v^{*}(1-\lambda t)} y,} \\
\psi & =\sqrt{\frac{v^{*} c}{(1-\lambda t)} \times f(\eta),} \\
\theta(\eta) & =\frac{T-T_{\infty}}{T_{w}-T_{\infty}} \\
\phi(\eta) & =\frac{C-C_{\infty}}{C_{w}-C_{\infty}}
\end{aligned}
$$


and the relations given by (14):

$$
\begin{aligned}
& T(x, t)=T_{\infty}+\frac{b x}{(1-\lambda t)^{2}} \theta(\eta) \\
& C(x, t)=C_{\infty}+\frac{b x}{(1-\lambda t)^{2}} \phi(\eta)
\end{aligned}
$$

where $v^{*}=\mu / \rho$ is the free stream kinematic viscosity, $\psi(x, y)$ is a stream function which defines the velocity components in the form $u=\partial \psi / \partial y=(c x /(1-\lambda t)) f^{\prime}(\eta), v=$ $-\partial \psi / \partial x=-\sqrt{\nu^{*} c /(1-\lambda t)} f(\eta)$ and it satisfies the continuity equation (1), $f(\eta)$ represents injection and suction, $\eta$ is the dimensionless space variable, and $\theta(\eta)$ and $\phi(\eta)$ are dimensionless of temperature and concentration of the fluid, respectively.

Following Hunegnaw and Kishan [21] and Vajravelu [30] the thermal conductivity of nanofluid $K(T)$ can vary linearly with temperature via a function shown in (15):

$$
K(T)=K_{\infty}\left(1+\frac{\beta_{1}}{\Delta T}\left(T-T_{\infty}\right)\right)
$$

and in terms of dimensionless temperature equation (15) reducing to equation (16):

$$
K(\theta)=K_{\infty}\left(1+\beta_{1} \theta\right)
$$

where $K(\theta)$ is the variation thermal conductivity with respect to dimensionless temperature, $K_{\infty}$ is the thermal conductivity of the fluid far away from the heated sheet, and $\beta_{1}$ is a small parameter that depends on the nature of the fluid and it measures the rate of change of thermal conductivity with temperature.

In view of several analogies between heat and mass transfer during cooling process and following Dimian and Hadhoda [20], one can model the diffusion coefficient $D(T)$ as a linear function of temperature shown in equation (17):

$$
D(T)=D_{\infty}\left(1+\frac{\beta_{2}}{\Delta T}\left(T-T_{\infty}\right)\right)
$$

Again as before, (17) may also be written in terms of dimensionless temperature as shown in

$$
D(\theta)=D_{\infty}\left(1+\beta_{2} \theta\right)
$$

where $D(\theta)$ is the variation diffusion coefficient with respect to dimensionless temperature, $D_{\infty}$ is the diffusion coefficient of the fluid far away from the heated sheet and $\beta_{2}$ is a small parameter that depends on the nature of the fluid and it measures the rate of change of chemical diffusivity with temperature.
Using equations (12)-(14), (16) and (18), in equations (2), (4), (11) and (5) the following ordinary differential equations (19) to (21) are obtained:

$$
\begin{aligned}
& f^{\prime \prime \prime}=A \frac{\eta}{2} f^{\prime \prime}+[A+\varphi+M] f^{\prime}+\left(f^{\prime}\right)^{2}-f f^{\prime \prime}-G_{r} \theta-G_{c} \phi \\
& \theta^{\prime \prime} \\
& =\frac{-\beta_{1}\left(\theta^{\prime}\right)^{2}+P_{r}\left[A(\eta / 2) \theta^{\prime}+2 A \theta+f^{\prime} \theta-f \theta^{\prime}-E_{c}\left(f^{\prime \prime}\right)^{2}\right]}{\left(1+R+\beta_{1} \theta\right)} \\
& \phi^{\prime \prime}=\frac{-\beta_{2}\left(\phi^{\prime} \theta^{\prime}\right)+S_{c}\left[A(\eta / 2) \phi^{\prime}+2 A \phi+K_{r} \phi+f^{\prime} \phi-f \phi^{\prime}\right]}{\left(1+\beta_{2} \theta\right)}
\end{aligned}
$$

with the corresponding initial and boundary conditions in the dimensionless form given by equation (22):

$$
\begin{aligned}
f(0) & =f_{w}, \\
f^{\prime}(0) & =1, \\
\theta(0) & =1, \\
\phi(0) & =1 \\
f^{\prime}(\eta) & \longrightarrow 0, \\
\theta(\eta) & \longrightarrow 0, \\
\phi(\eta) & \longrightarrow 0
\end{aligned}
$$

where $A, \varphi, M, G_{r}, G_{c}, P_{r}, R, K_{r}, E_{c}$, and $S_{c}$ are the unsteadiness parameter, porous medium parameter, magnetic parameter, thermal Grashof number, solutal or concentration Grashof number, Prandtl number, thermal radiation parameter, chemical reaction parameter, Eckert number, and the Schmidt number, respectively, and are defined in

$$
\begin{aligned}
A & =\frac{\lambda}{c}, \\
\varphi & =\frac{\nu^{*}(1-\lambda t)}{\kappa^{*} c}, \\
M & =\frac{\sigma B_{0}^{2}(1-\lambda t)}{\rho c}, \\
G_{r} & =\frac{g \beta_{T} x\left(T_{w}-T_{\infty}\right)}{u_{w}^{2}} \cos \alpha, \\
S_{c} & =\frac{\nu^{*}}{D_{\infty}} \\
G_{c} & =\frac{g \beta_{c} x\left(C_{w}-C_{\infty}\right)}{u_{w}^{2}} \cos \alpha, \\
P_{r} & =\frac{C_{p} \mu}{K_{\infty}},
\end{aligned}
$$




$$
\begin{aligned}
R & =\frac{16 T_{\infty}^{3} \sigma^{*}}{3 k_{1} K_{\infty}}, \\
K_{r} & =\frac{K_{c}(1-\lambda t)}{c}, \\
E_{c} & =\frac{u_{w}^{2}}{C_{p}\left(T_{w}-T_{\infty}\right)},
\end{aligned}
$$

Note further the prime symbol denoting the differentiation with respect to dimensionless space variable $(\eta)$. In this model the quantities of physical and practical engineering interest are the skin friction coefficient $\left(C_{f}\right)$, the Nusselt number $\left(N u_{x}\right)$, and the Sherwood number $\left(S h_{x}\right)$ which are defined in (24):

$$
\begin{aligned}
C_{f} & =\frac{\tau_{w}}{(1 / 2) \rho u_{w}^{2}}, \\
N u_{x} & =\frac{x q_{w}}{K\left(T_{w}-T_{\infty}\right)}, \\
S h_{x} & =\frac{x h_{m}}{D\left(C_{w}-C_{\infty}\right)}
\end{aligned}
$$

In which, $\tau_{w}, q_{w}$, and $h_{m}$ are the skin friction or shear stress, heat flux, and mass flux from the sheet and are, respectively, given in (25):

$$
\begin{aligned}
& \tau_{w}=\mu\left[\frac{\partial u}{\partial y}\right]_{y=0}, \\
& q_{w}=-K\left[\frac{\partial T}{\partial y}\right]_{y=0}, \\
& h_{m}=-D\left[\frac{\partial C}{\partial y}\right]_{y=0}
\end{aligned}
$$

Substitutions of (13) and the relation (14) into (24) transform the skin friction, heat flux, and mass flux into (26) to (28), respectively:

$$
\begin{aligned}
f^{\prime \prime}(0) & =\frac{1}{2} \sqrt{R e_{x}} C_{f} \\
-\theta^{\prime}(0) & =\frac{N u_{x}}{\sqrt{R e_{x}}} \\
-\phi^{\prime}(0) & =\frac{S h_{x}}{\sqrt{R e_{x}}}
\end{aligned}
$$

where $f^{\prime \prime}(0),-\theta^{\prime}(0)$, and $-\phi^{\prime}(0)$ are the skin friction, Nusselt number, and Sherwood number, respectively; note further that $R e_{x}=u_{w} x / v^{*}=c x^{2} / \nu^{*}(1-\lambda t)$ is the local Reynolds number.

\section{Numerical Solution of the Problem}

In order to solve the system of ordinary differential equations (19)-(21) with their corresponding initial and boundary conditions (22) numerically, the domain $[0, \infty)$ has been substituted by the bounded domain $\left[0, \eta_{\infty}\right]$ where $\eta_{\infty}$ is a suitable finite real number that should be chosen in such a way that the solution satisfies the domain. Also (19)-(21) form a highly nonlinear coupled initial boundary value problem of third and second order ODEs. For this reason, (19)-(21) have been reduced to a system of seven initial problems of the first order of seven unknowns following the supposition in

$$
\begin{aligned}
f & =y_{1}, \\
f^{\prime} & =y_{2}, \\
f^{\prime \prime} & =y_{3}, \\
\theta & =y_{4}, \\
\theta^{\prime} & =y_{5}, \\
\phi & =y_{6}, \\
\phi^{\prime} & =y_{7},
\end{aligned}
$$

Thus we develop the most effective numerical shooting technique in line with the fourth order Runge-Kutta scheme. To solve this system, we require seven initial conditions whereas we have only four initial conditions for $f(0), f^{\prime}(0), \theta(0)$, and $\phi(0)$, while the other three $f^{\prime \prime}(0), \theta^{\prime}(0)$, and $\phi^{\prime}(0)$ were not given; hence, we employ numerical shooting technique where these three initial conditions are guessed to produce the required three ending boundary conditions. The step size $\Delta \eta=0.001$ is used to obtain the numerical solution with eight decimals $\left(1 \times 10^{-8}\right)$ as a criterion of convergence.

\section{Results and Discussion}

Equations (19) to (21) with the corresponding initial and boundary conditions (22) have been solved numerically using the Runge-Kutta scheme together with shooting technique. The influence of the nondimensional governing parameters on velocity, temperature, concentration fields as well as friction factor, Nusselt number, and Sherwood number has been established by assigning some numerical values to the nondimensional parameters. The results obtained are discussed and presented through graphs and tables.

The nondimensional governing parameters in this research are suction/injection parameter $\left(f_{w}\right)$, unsteadiness parameter $(A)$, porous medium parameter $(\varphi)$, magnetic parameter $(M)$, the thermal Grashof number $\left(G_{r}\right)$, the solutal or concentration Grashof number $\left(G_{c}\right)$, variable thermal conductivity parameter $\left(\beta_{1}\right)$, variable diffusion coefficient parameter $\left(\beta_{2}\right)$, the Prandtl number $\left(P_{r}\right)$, the thermal radiation parameter $(R)$, the chemical reaction parameter $\left(K_{r}\right)$, the Eckert number $\left(E_{c}\right)$, and Schmidt number $\left(S_{c}\right)$.

With an exceptional of the varied parameters in Table 1 and on Figures 2-23 the values of these nondimensional parameters are considered to be constant throughout this study with their values $f_{w}=0.1, A=0.5, \varphi=U=0.5, M=$ $0.5, G_{r}=0.1, G_{c}=0.1, \beta_{1}=0.012, \beta_{2}=0.01, P_{r}=0.72, R=$ $0.01, K_{r}=0.1, E_{c}=0.03$, and $S_{c}=2$. To be realistic, the values 
TABle 1: Comparison of $-\theta^{\prime}(0)$ when values of $\varphi=0, M=0, G_{c}=0, \beta_{1}=0, \beta_{2}=0, R=0, K_{r}=0, E_{c}=0$, and $S_{c}=0$.

\begin{tabular}{|c|c|c|c|c|c|c|}
\hline$A$ & $f_{w}$ & $P_{r}$ & $G_{r}$ & Ishak et al. (2009) [31] & Sandeep et al. (2015) [32] & Present Study \\
\hline 0 & -1.5 & 0.72 & 0 & 0.4570 & 0.4566 & 0.4570 \\
\hline 0 & -1.5 & 1 & 0 & 0.5000 & 0.5001 & 0.5001 \\
\hline 0 & -1.5 & 10 & 0 & 0.6452 & 0.6451 & 0.6451 \\
\hline 0 & 0 & 0.01 & 0 & 0.0197 & 0.0192 & 0.0197 \\
\hline 0 & 0 & 0.72 & 0 & 0.8086 & 0.8082 & 0.8086 \\
\hline 0 & 0 & 1 & 0 & 1.0000 & 1.0001 & 1.0000 \\
\hline 0 & 0 & 3 & 0 & 1.9237 & 1.9231 & 1.9239 \\
\hline 0 & 0 & 10 & 0 & 3.7207 & 3.7202 & 3.7207 \\
\hline 0 & 1.5 & 0.72 & 0 & 1.4944 & 1.4945 & 1.4944 \\
\hline 0 & 1.5 & 1 & 0 & 2.0000 & 2.0001 & 2.0000 \\
\hline 0 & 1.5 & 10 & 0 & 16.0842 & 16.0837 & 16.0842 \\
\hline 1 & 0 & 1 & 0 & 1.6820 & --- & 1.6820 \\
\hline 1 & 0 & 1 & 1 & 1.7039 & --- & 1.7037 \\
\hline
\end{tabular}

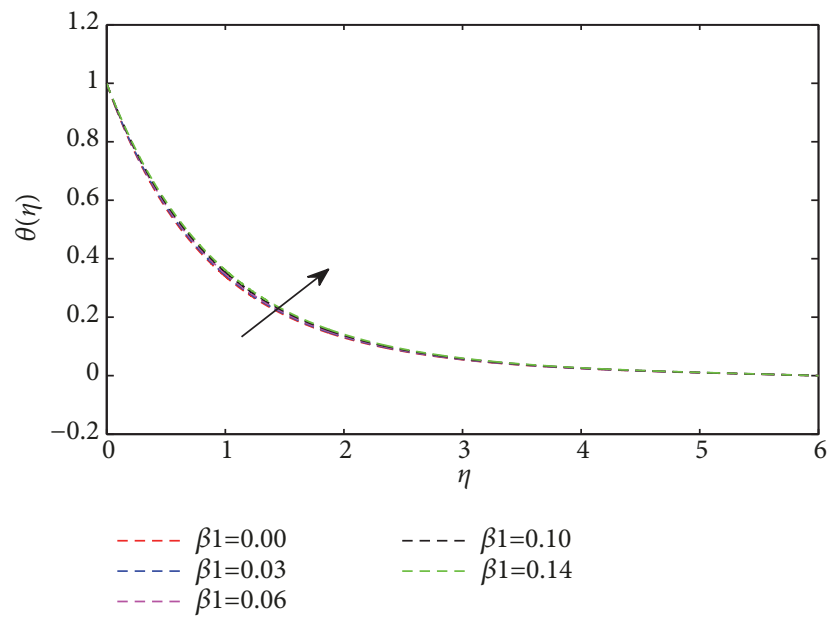

FIGURE 2: Effects of variable thermal conductivity on temperature profiles.

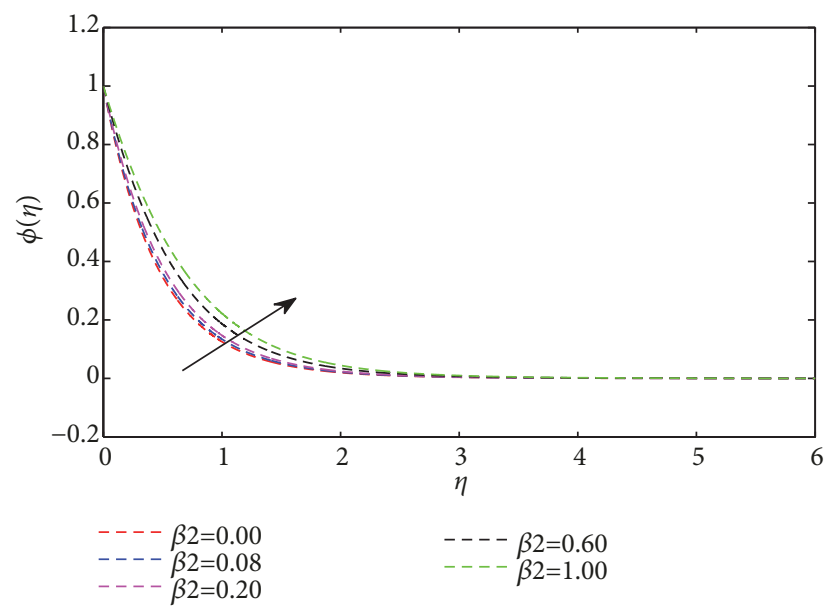

FIGURE 3: Effects of variable diffusion coefficient on concentration profiles.

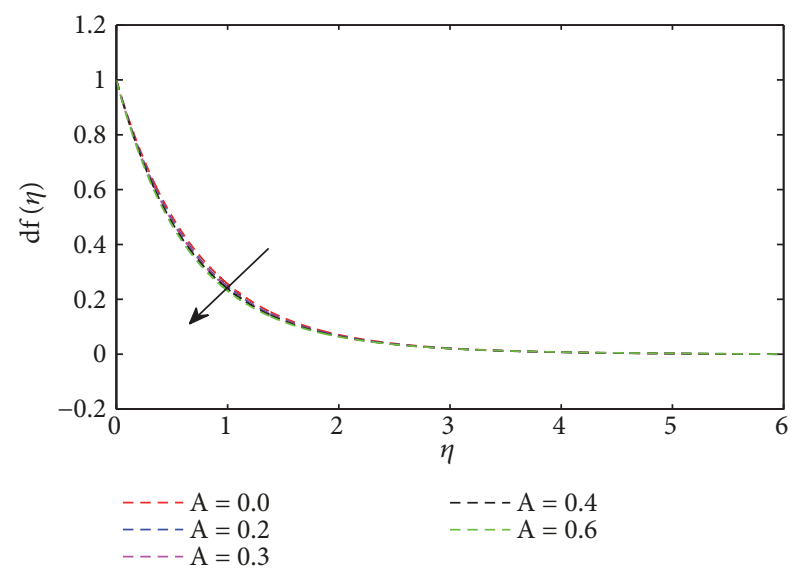

FIGURE 4: Effects of unsteadiness parameter on velocity profiles.

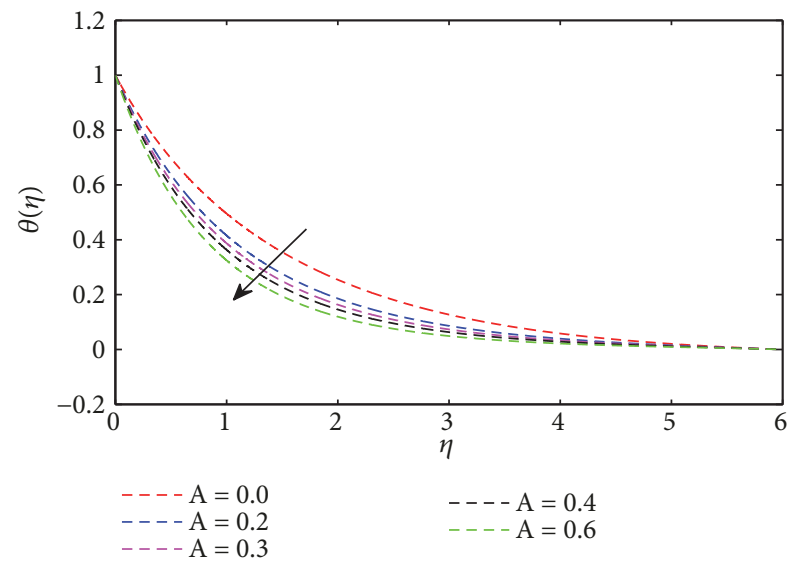

FIGURE 5: Effects of unsteadiness parameter on temperature profiles.

of Prandtl number $\left(P_{r}\right)$ are chosen as $P_{r}=0.60,0.71$ and 1.00 at $4^{\circ} \mathrm{C}$ which corresponds to water vapour, air and electrolytic solution, respectively, and the values of Schmidt number $\left(S_{c}\right)$ are chosen as $S_{c}=0.22,0.60,0.78,1.00$ and 2.62 which 


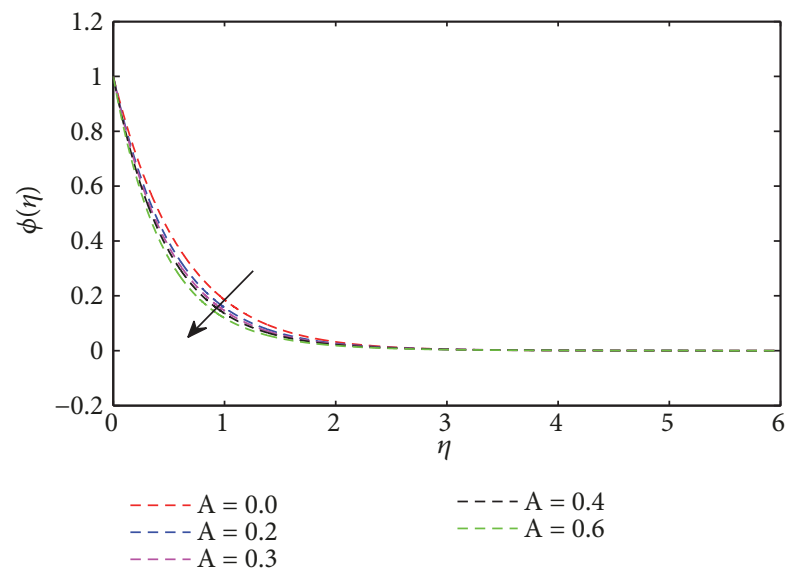

FIGURE 6: Effects of unsteadiness parameter on concentration profiles.

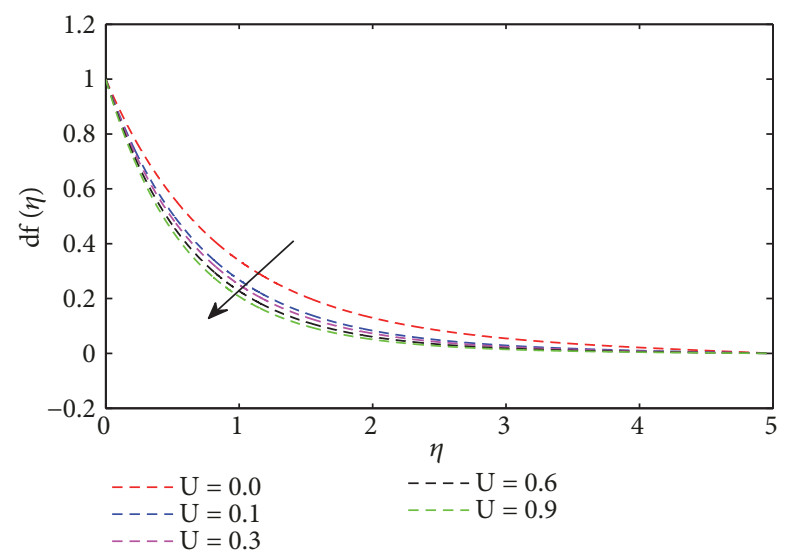

FIgURE 7: Effects of porous medium parameter $(U)$ on velocity profiles.

corresponds to hydrogen, water vapour, ammonia, methanol and propyl benzene at $20^{\circ} \mathrm{C}$, respectively, as presented by Shanker et al. [33].

In order to check the validity and accuracy of the present analysis, the results for heat transfer $-\theta^{\prime}(0)$ are compared with those reported by Ishak et al. [31] and Sandeep et al. [32] as shown in Table 1 and a good agreement has been observed.

Table 2 presents the effects of nondimensional governing parameters on the skin friction, Nusselt and Sherwood numbers. It is evident from the table that an increase in porosity parameter and magnetic field reduces the friction factor, heat and mass transfer rates. The increase in both thermal conductivity and radiative heat flux parameters decreases the heat transfer rate but increases the friction factor and mass transfer rates. Also the increase in thermal and concentration Grashof number increases the friction factor, heat and mass transfer rates.

Note further from Table 2 that the increase in diffusion coefficient results in the increase in skin friction and heat transfer, but it decreases the mass transfer rate. Increase in unsteadiness parameter causes the increase in both heat and mass transfer rate whereas it causes a decrease in skin friction.

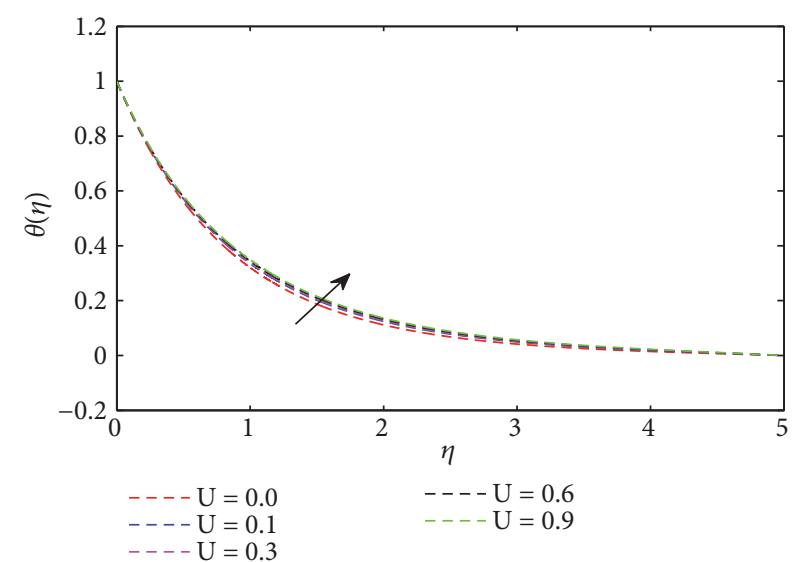

FIGURE 8: Effects of porous medium parameter $(U)$ on temperature profiles.

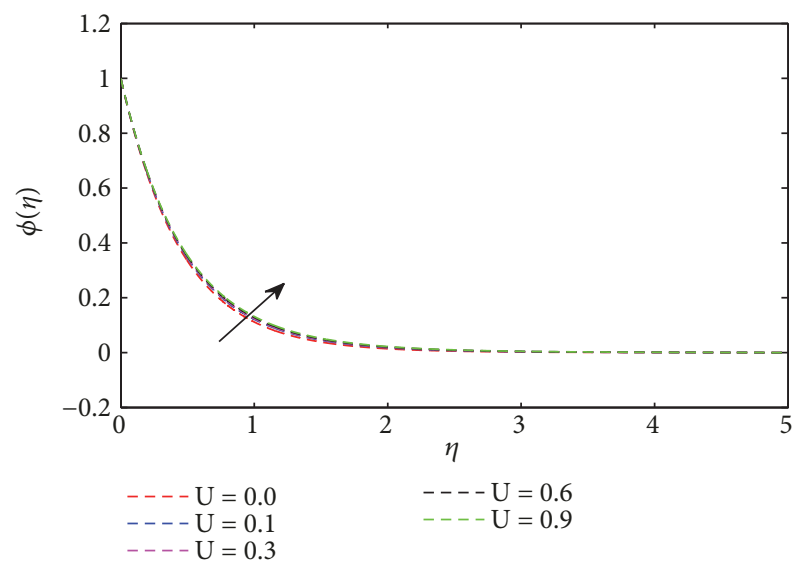

FIGURE 9: Effects of porous medium parameter $(U)$ on concentration profiles.

In addition to this, as the chemical reaction parameter increases, it reduces the skin friction and heat transfer, but the mass transfer rate increases.

The trends of dimensionless velocity $f^{\prime}(\eta)$, temperature $\theta(\eta)$, and concentration $\phi(\eta)$ profiles for the different values of flow parameters are shown graphically in Figures 2-23.

The effect of thermal conductivity parameter $\left(\beta_{1}\right)$ on the temperature is shown in Figure 2. It can be noted that the temperature tends to increase as the variable thermal conductivity parameter increases. This is due to the increase in thermal boundary layer thickness; its effect is higher at the boundary layer and decreases as you approach the free stream.

Figure 3 depicts the effect of variable diffusion coefficient parameter $\left(\beta_{2}\right)$ on concentration profile. Meanwhile, the diffusion coefficient $D(T)$ is a linear function of temperature; it follows that as temperature increases, the diffusion coefficient increases and, consequently, the concentration tends to increase. This is due to the Fick's law of concentration which shows that the diffusion coefficient is very much depending on the concentration gradient. Thus, as $\beta_{2}$ increases, the concentration increases. 
TABLE 2: The values of $f^{\prime \prime}(0),-\theta^{\prime}(0)$, and $-\phi^{\prime}(0)$ for different values of $\beta_{1}, \beta_{2}, A, \varphi, M, G_{r}, G_{c}$, $K_{r}$, and $R$ when the values of the parameters $f_{w}=0.1, P_{r}=0.72, E_{c}=0.03$, and $S_{c}=2$.

\begin{tabular}{|c|c|c|c|c|c|c|c|c|c|c|c|}
\hline$\beta_{1}$ & $\beta_{2}$ & $A$ & $\varphi$ & $M$ & $G_{r}$ & $G_{c}$ & $K_{r}$ & $R$ & $f^{\prime \prime}(0)$ & $-\theta^{\prime}(0)$ & $-\phi^{\prime}(0)$ \\
\hline 0.020 & 0.01 & 0.5 & 0.5 & 0.5 & 0.1 & 0.1 & 0.1 & 0.01 & -1.5222 & 1.1281 & 2.1268 \\
\hline 0.035 & 0.01 & 0.5 & 0.5 & 0.5 & 0.1 & 0.1 & 0.1 & 0.01 & -1.5221 & 1.1167 & 2.1269 \\
\hline 0.040 & 0.01 & 0.5 & 0.5 & 0.5 & 0.1 & 0.1 & 0.1 & 0.01 & -1.5221 & 1.1129 & 2.1269 \\
\hline 0.012 & 0.00 & 0.5 & 0.5 & 0.5 & 0.1 & 0.1 & 0.1 & 0.01 & -1.5224 & 1.1344 & 2.1408 \\
\hline 0.012 & 0.08 & 0.5 & 0.5 & 0.5 & 0.1 & 0.1 & 0.1 & 0.01 & -1.5217 & 1.1345 & 2.0358 \\
\hline 0.012 & 0.30 & 0.5 & 0.5 & 0.5 & 0.1 & 0.1 & 0.1 & 0.01 & -1.5201 & 1.1347 & 1.8015 \\
\hline 0.012 & 0.01 & 0.1 & 0.5 & 0.5 & 0.1 & 0.1 & 0.1 & 0.01 & -1.4117 & 0.8458 & 1.7363 \\
\hline 0.012 & 0.01 & 0.3 & 0.5 & 0.5 & 0.1 & 0.1 & 0.1 & 0.01 & -1.4685 & 1.0047 & 1.9424 \\
\hline 0.012 & 0.01 & 0.5 & 0.1 & 0.5 & 0.1 & 0.1 & 0.1 & 0.01 & -1.3833 & 1.1489 & 2.1447 \\
\hline 0.012 & 0.01 & 0.5 & 0.3 & 0.5 & 0.1 & 0.1 & 0.1 & 0.01 & -1.4544 & 1.1414 & 2.1432 \\
\hline 0.012 & 0.01 & 0.5 & 0.5 & 0.5 & 0.1 & 0.1 & 0.1 & 0.01 & -1.5223 & 1.1344 & 2.1269 \\
\hline 0.012 & 0.01 & 0.5 & 0.5 & 0.1 & 0.1 & 0.1 & 0.1 & 0.01 & -1.3833 & 1.1489 & 2.1432 \\
\hline 0.012 & 0.01 & 0.5 & 0.5 & 0.3 & 0.1 & 0.1 & 0.1 & 0.01 & -1.4544 & 1.1414 & 2.1347 \\
\hline 0.012 & 0.01 & 0.5 & 0.5 & 0.5 & 0.1 & 0.1 & 0.1 & 0.01 & -1.5223 & 1.1344 & 2.1269 \\
\hline 0.012 & 0.01 & 0.5 & 0.5 & 0.5 & 0.1 & 0.1 & 0.1 & 0.01 & -1.5223 & 1.1344 & 2.1269 \\
\hline 0.012 & 0.01 & 0.5 & 0.5 & 0.5 & 0.3 & 0.1 & 0.1 & 0.01 & -1.4459 & 1.1431 & 2.1364 \\
\hline 0.012 & 0.01 & 0.5 & 0.5 & 0.5 & 0.5 & 0.1 & 0.1 & 0.01 & -1.3706 & 1.1515 & 2.1455 \\
\hline 0.012 & 0.01 & 0.5 & 0.5 & 0.5 & 0.1 & 0.1 & 0.1 & 0.01 & -1.5223 & 1.1344 & 2.1269 \\
\hline 0.012 & 0.01 & 0.5 & 0.5 & 0.5 & 0.1 & 0.3 & 0.1 & 0.01 & -1.4681 & 1.1389 & 2.1323 \\
\hline 0.012 & 0.01 & 0.5 & 0.5 & 0.5 & 0.1 & 0.5 & 0.1 & 0.01 & -1.4143 & 1.1433 & 2.1376 \\
\hline 0.012 & 0.01 & 0.5 & 0.5 & 0.5 & 0.1 & 0.1 & 0.1 & 0.01 & -1.5223 & 1.1344 & 2.1269 \\
\hline 0.012 & 0.01 & 0.5 & 0.5 & 0.5 & 0.1 & 0.1 & 0.4 & 0.01 & -1.5235 & 1.1342 & 2.2785 \\
\hline 0.012 & 0.01 & 0.5 & 0.5 & 0.5 & 0.1 & 0.1 & 0.8 & 0.01 & -1.5249 & 1.1340 & 2.4630 \\
\hline 0.012 & 0.01 & 0.5 & 0.5 & 0.5 & 0.1 & 0.1 & 0.1 & 0.20 & -1.5206 & 1.0296 & 2.1274 \\
\hline 0.012 & 0.01 & 0.5 & 0.5 & 0.5 & 0.1 & 0.1 & 0.1 & 0.40 & -1.5190 & 0.9440 & 2.1278 \\
\hline 0.012 & 0.01 & 0.5 & 0.5 & 0.5 & 0.1 & 0.1 & 0.1 & 0.50 & -1.5183 & 0.9080 & 2.1280 \\
\hline
\end{tabular}

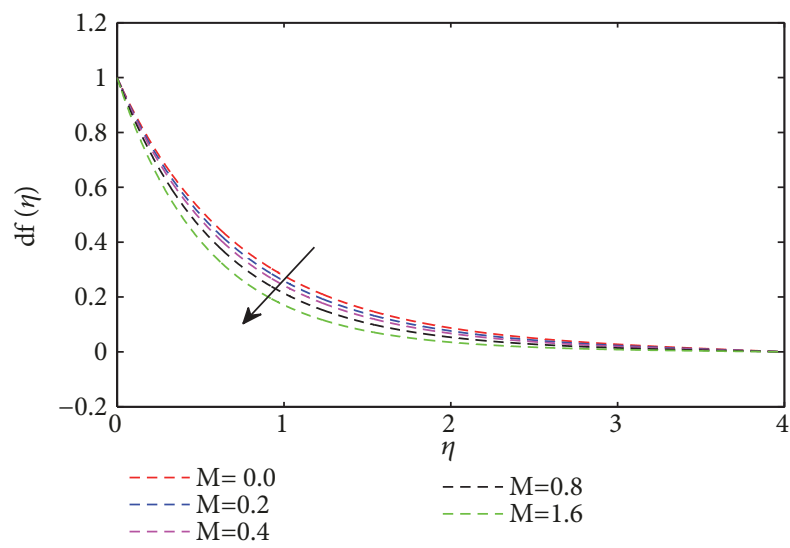

FIGURE 10: Effects of magnetic parameter $(M)$ on velocity profiles.

Figures 4, 5, and 6 present the typical effects of unsteadiness parameter $(A)$ on the velocity, temperature, and concentration profiles, respectively; it can be noted that an increase in $A$ results in the decrease in velocity, temperature, and concentration profiles of the nanofluid. Generally, as $A$ increases it causes thickening of the thermal boundary layer.

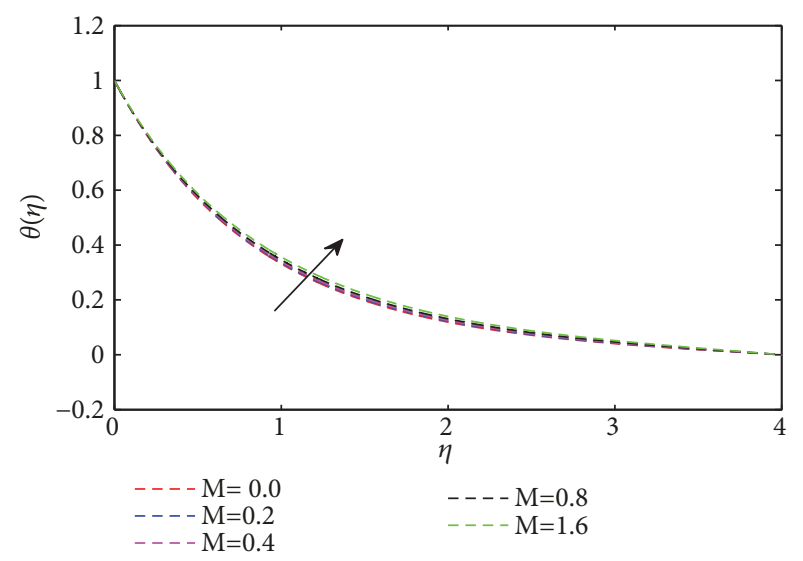

FIgURE 11: Effects of magnetic parameter $(M)$ on temperature profiles.

This effect causes the dimensionless velocity, temperature, and concentration to decrease.

The effects of the porous medium parameter $(\varphi)$ on velocity, temperature, and concentration are illustrated in Figures 7-9, respectively. It can be noted from Figure 7 that 


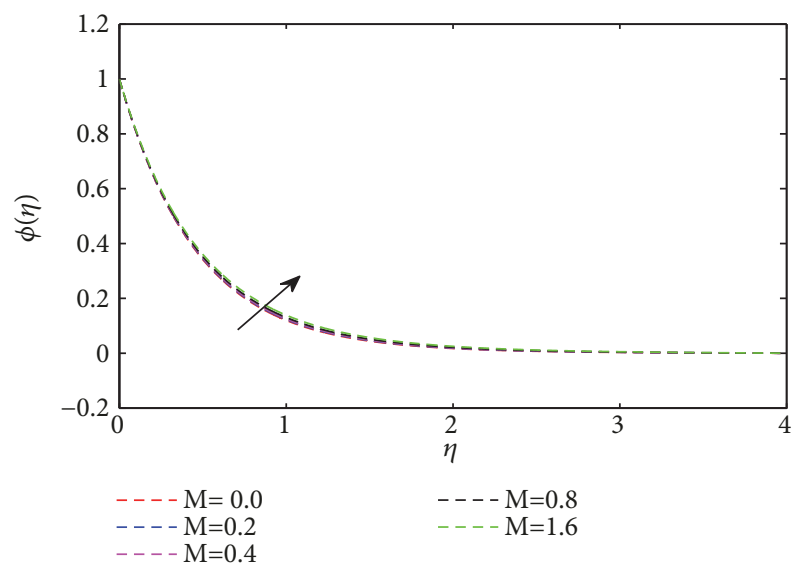

FIGURE 12: Effects of magnetic parameter $(M)$ on concentration profiles.

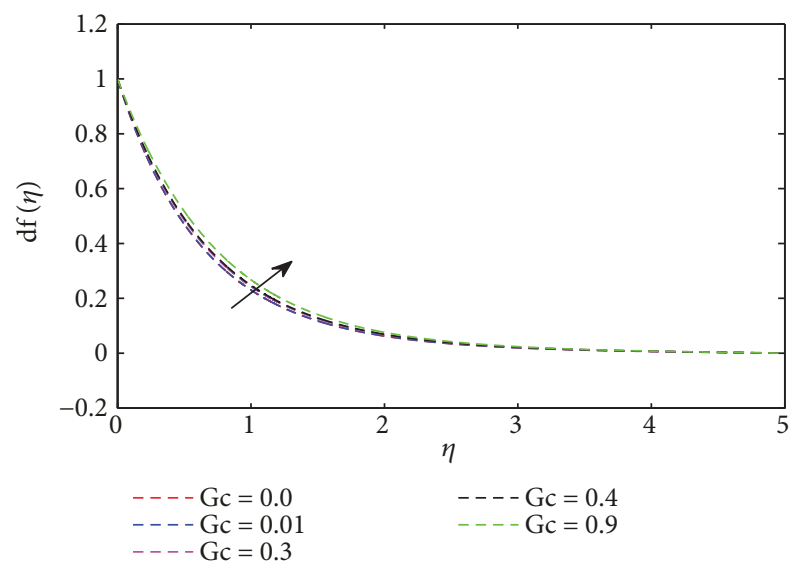

FIGURE 13: Effects of concentration Grashof number on velocity profiles.

as $\varphi$ increases, the dimensionless velocity decreases; this is because as the tightness of the porous medium parameter increases, the resistance against the flow increases; as a result the fluid velocity decreases and thus the heat is transferred from the hot sheet to the fluid and eventually the temperature and concentration of the fluid increase as shown in Figures 8 and 9.

Figure 10 presents the effects of external magnetic field $(M)$ on the velocity profile. It can be noted that as $M$ increases, the fluid velocity declines; this is due to the presence of transverse magnetic fields which causes the emergency of drag force (Lorentz force) opposing the motion of the nanofluid and as a results it retards the flow velocity. Figures 11 and 12 reveal that as $M$ increases, it improves the temperature and concentration profile. This is due to higher heat transfer from the hot sheet to the nanofluid and hence the fluid cools the sheet by absorbing its temperature.

Figure 13 displays the effect of concentration Grashof number $\left(G_{c}\right)$. It can be noted that an increase in $G_{c}$ results in the increase in the flow field. Since the concentration Grashof number is the ratio of thermal buoyancy force to the hydrodynamic force and happens on the boundary layer

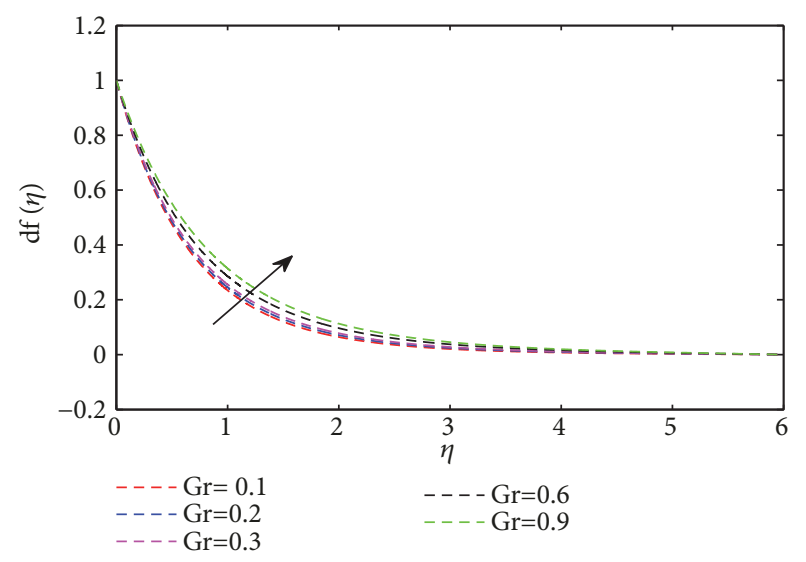

FIGURE 14: Effects of thermal Grashof number on velocity profiles.

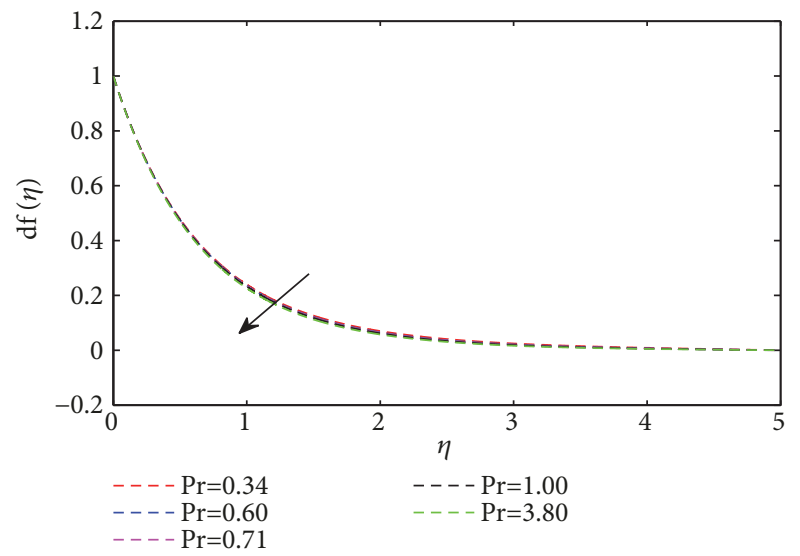

Figure 15: Effects of Prandtl number on velocity profiles.

due to variations of concentrations, an increase in thermal buoyancy effect on the nanofluid causes the fluid to cool the hot sheet.

Figure 14 presents the effect of thermal Grashof number $\left(G_{r}\right)$. It can be noted that an increase in $G_{r}$ results in the increase of nanofluid velocity. This is based on the fact that the thermal Grashof number is the ratio of thermal buoyancy force to the hydrodynamic force and happens on the boundary layer due to variations of temperature and thus an increase in thermal buoyancy effect on the nanofluid causes the said fluid to cool the hot sheet.

The effect of Prandtl number $\left(P_{r}\right)$ on dimensionless velocity is illustrated in Figure 15; it can be noted that as $P_{r}$ increases, the velocity of the flow field decreases and hence it causes the thermal boundary layer to shrink. It is interesting to note from Figure 16 that as $P_{r}$ increases, it causes the decrease in temperature of nanofluid; this is due to the fact that $P_{r}$ is inversely proportional to the thermal diffusion and directly proportional to the momentum diffusivity and thus as $P_{r}$ increases, it leads to the decrease in thermal diffusion and consequently the temperature and concentration of the fluid decreases.

Figure 17 depicts the effect of Schmidt number on concentration profile. It has been noted that the concentration 


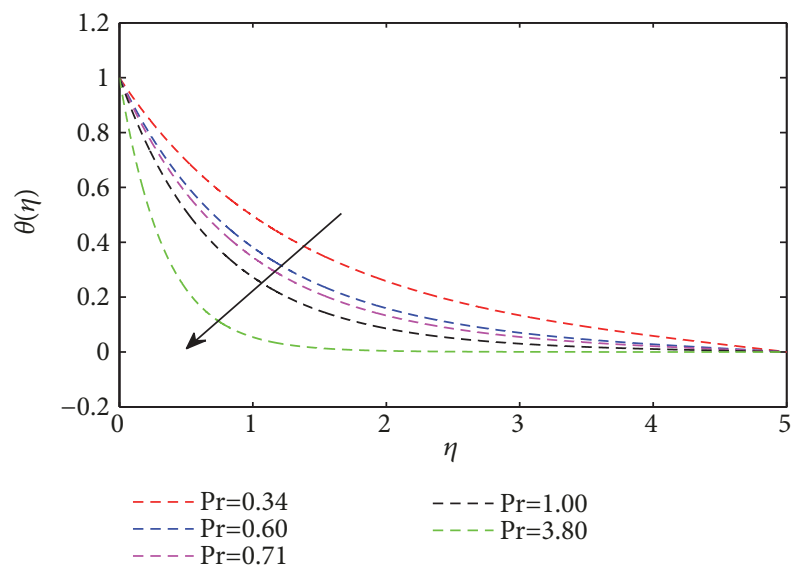

Figure 16: Effects of Prandtl number on temperature profiles.

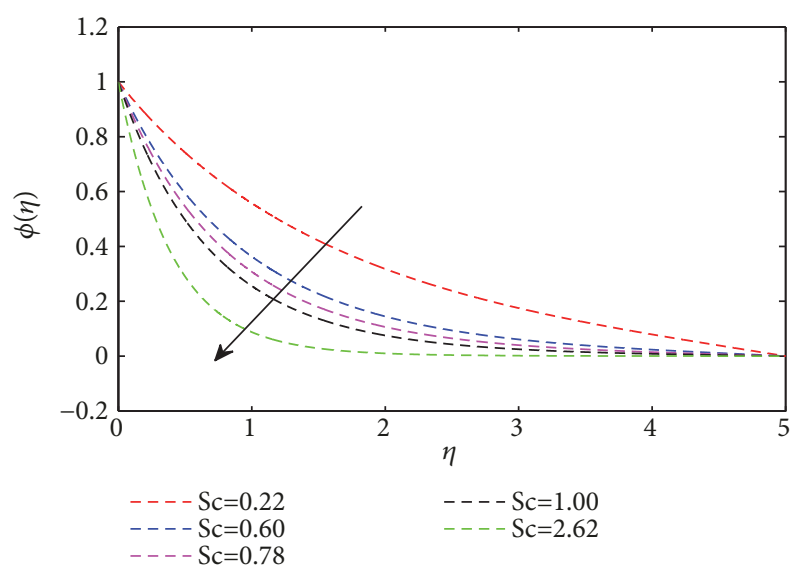

FIGURE 17: Effects of Schmidt number on concentration profiles.

of the fluid decreases as the Schmidt number $\left(S_{c}\right)$ increases; this is due to the fact that $S_{c}$ is directly proportional to the kinematic viscosity $S_{c}$ and inversely proportional to the mass diffusion coefficient $(D)$; thus, as $S_{c}$ increases, the mass diffusion coefficient $D$ decreases and as a result the diffusion foreign species become heavier and normally heavier species have higher $S_{c}$.

The effects of chemical reaction parameter $\left(K_{r}\right)$ on the dimensionless concentration profile are illustrated in Figure 18. It is clear from Figure 18 that as $K_{r}$ increases, the concentration profile decreases; this means $K_{r}$ is a slowing agent because it reduces the concentration in the boundary layer and hence at the point very near to the sheet the solute boundary layer turns out to be thinner and increases the mass transfer.

Figure 19 shows the effects of radiative heat flux parameter $(R)$ on the temperature profile. Generally, the presence of chemical reaction results in generations of the radiative heat flux and thus the increase in $R$ leads to the increase in the boundary layer thickness and improves the heat transfer rate on the surface and as a result the temperature distribution in the flow region increases as $R$ increases.

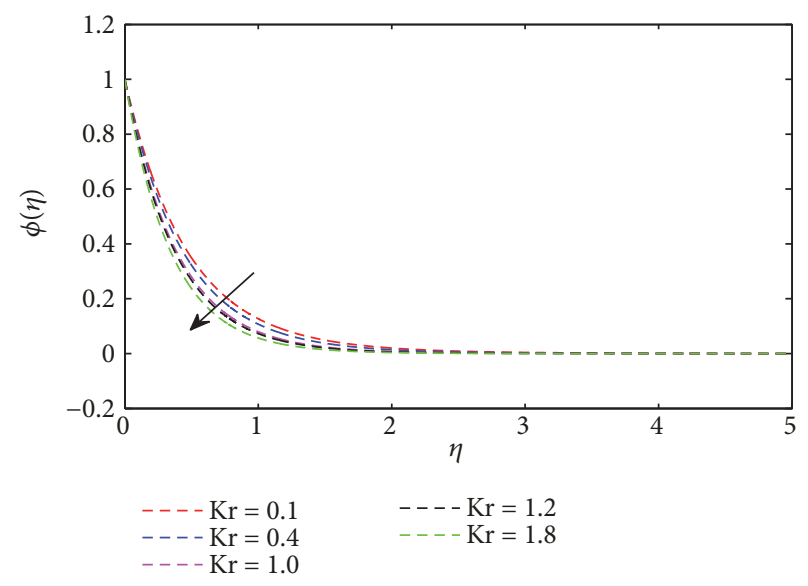

FIGURE 18: Effects of chemical reaction on concentration profiles.

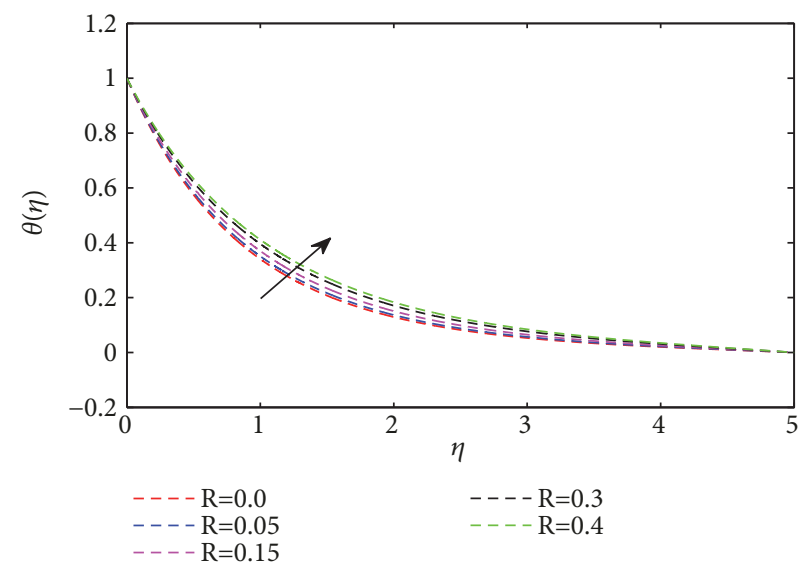

FIGURE 19: Effects of thermal radiation parameter on temperature profiles.

Figure 20 depicts the effects of inclination angles $(\alpha)$ on the velocity profile. It has been observed that as the inclination angle increases in the anticlockwise from vertical axis, the velocity of nanofluids decreases. This is due to the fact that as the inclination angle increases, it causes both concentration Grashof number $\left(G_{c}\right)$ and thermal Grashof number $\left(G_{r}\right)$ to decrease and thus the thermal buoyancy effect on the nanofluid decreases and finally limits the cooling process.

The effects of suction/injection parameter $f_{w}$ on dimensionless velocity, temperature, and concentration are presented in Figures 21-23. The increase in $f_{w}$ within the porous retards the fluid velocity and hence it causes the fluid viscosity to increase, as a result the dimensionless temperature and concentration decrease. This is because more fluids are forced to pass through the permeable sheet and as a result the fluids that are very close to the hot sheet suffer decrease in temperature.

\section{Conclusion}

In this research, a numerical analysis on unsteady MHD flow of nanofluid with variable properties over an inclined 


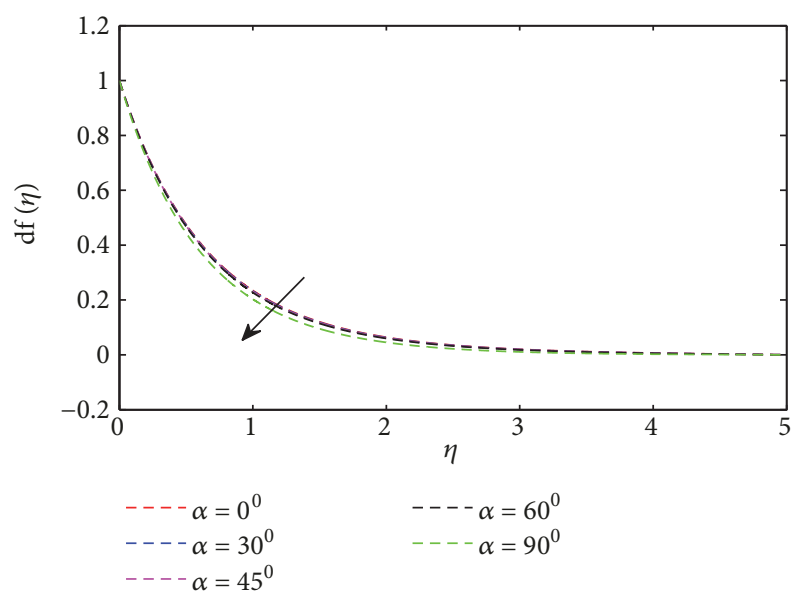

Figure 20: Effects of inclination angles on velocity profile.

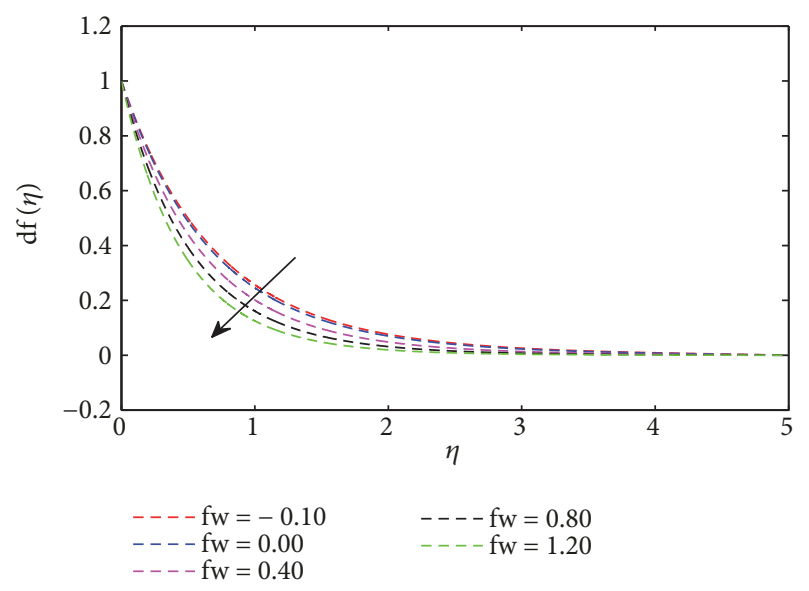

FIGURE 21: Effects of suction/injection parameter on velocity profiles.

stretching sheet in the presence of thermal radiation and chemical reaction is presented. The influence of the nondimensional governing parameters such as suction/injection, unsteadiness, porous medium, magnetic parameter, thermal Grashof number, concentration Grashof number, variable thermal conductivity, variable diffusion coefficient, Prandtl number, thermal radiation, the chemical reaction, Eckert and Schmidt number on the velocity, temperature, concentration fields as well as skin friction, and Nusselt and Sherwood number has been established.

It is found that an increase in variable thermal conductivity parameter results in the increase in temperature of the nanofluid. The increase in variable diffusion coefficient parameter results in the increase in concentration. The increase in either unsteadiness or suction parameter results in the decrease in the velocity, temperature, and concentration. The increase in Schmidt number or chemical reaction parameter results in the decrease in concentration. The increase in Prandtl number results in a decrease in both velocity and temperature. The increase in either concentration or thermal Grashof number results in the increase in velocity.

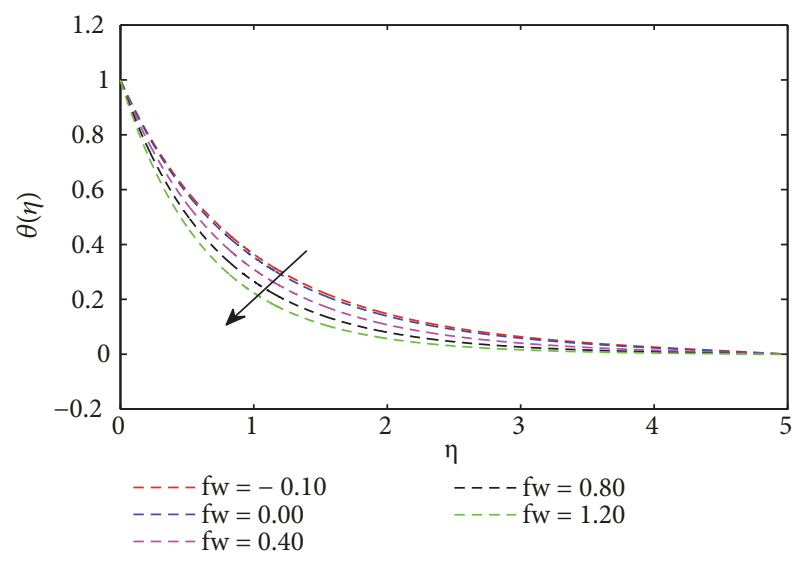

FIGURE 22: Effects of suction/injection parameter on temperature profiles.

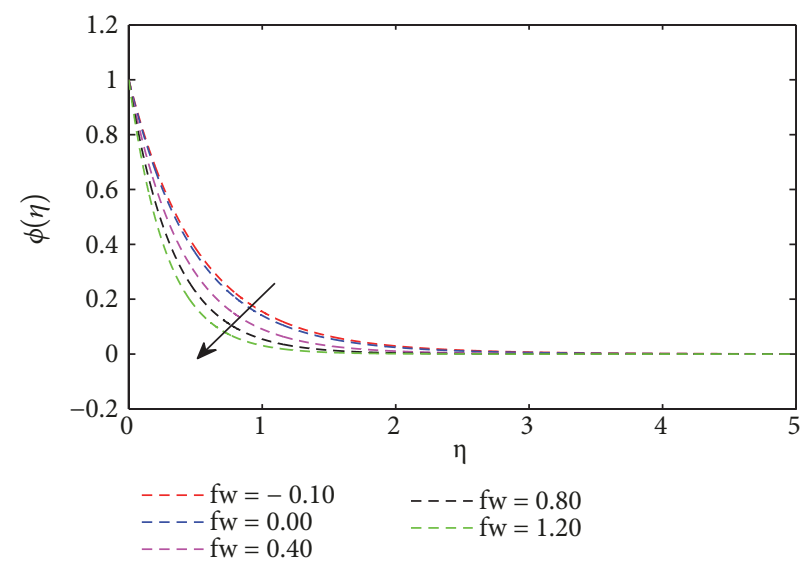

FIGURE 23: Effects of suction/injection parameter on concentration profiles.

The increase in thermal radiation parameter results in the increase in temperature.

It is further observed that porosity and magnetic parameters have the ability of reducing the nanofluid velocity and enhancing the temperature and concentration profiles and also the duo has the capability of reducing the skin friction coefficient, heat, and mass transfer rates.

\section{Nomenclature}

$x: \quad$ Coordinate along the stretching sheet

$y: \quad$ Distance normal to the stretching sheet

$c: \quad$ Initial stretching rate

b: $\quad$ Constants

$t$ : Time

$u$ : $\quad$ Velocity along the $x$ - direction

$v$ : $\quad$ Velocity along the $y$ - direction

$u_{w}(x, t)$ : Velocity of the stretching sheet

$v_{w}(t)$ : Velocity of the mass transfer

$F$ : $\quad$ Force applied along the $x$ - axis

$T_{w}(x, t)$ : Temperature of the sheet at the wall 


$\begin{array}{ll}C_{w}(x, t): & \text { Concentration of the fluid at the wall } \\ T_{\infty}: & \text { Uniform temperature far from the sheet } \\ C_{\infty}: & \text { Uniform concentration far from the sheet } \\ B_{0}: & \text { Magnetic induction } \\ C_{p}: & \text { Specific heat at constant pressure } \\ K_{r}: & \text { Chemical reaction parameter } \\ E_{c}: & \text { Eckert number } \\ S_{c}: & \text { Schmidt number } \\ C_{f}: & \text { Skin friction coefficient } \\ N u_{x}: & \text { Nusselt number } \\ S h_{x}: & \text { Sherwood number } \\ q_{w}: & \text { Heat flux } \\ h_{m}: & \text { Mass flux from the sheet } \\ R_{e_{x}}: & \text { Reynolds number } \\ K: & \text { Thermal conductivity } \\ D: & \text { Molecular diffusivity } \\ T: & \text { Temperature of the species } \\ C: & \text { Concentration of the species } \\ g: & \text { Acceleration due to gravity } \\ q_{r}: & \text { Radiation heat flux } \\ v_{0}: & \text { Constant } \\ k_{1}: & \text { Coefficient of absorption } \\ K_{\infty}: & \text { Thermal conductivity of the ambient } \\ D_{\infty}: & \text { Diffusion coefficient of the ambient } \\ M: & \text { Magnetic parameter } \\ A: & \text { Unsteadiness parameter } \\ G_{r}: & \text { Thermal Grashof number } \\ G_{c}: & \text { Solutal or concentration Grashof number } \\ P_{r}: & \text { Prandtl number } \\ R: & \text { Thermal Radiation parameter } \\ f_{w}: & \text { Suction/Injection parameter. } \\ & \end{array}$

\section{Greek Symbols}

$\alpha:$ Inclination angle
$\lambda:$ Constant
$\psi:$ Stream function
$\sigma^{*}:$ Stefan- Boltzmann constant
$\beta_{1}:$ Variable thermal conductivity
$\beta_{2}:$ Variable diffusion coefficient
$\tau_{w}:$ Shear stress
$\varphi:$ Porous medium parameter
$\Delta:$ Delta
$\kappa^{*}:$ Permeability
$\nu^{*}:$ Kinematic viscosity
$\mu:$ Coefficient of viscosity
$\phi:$ Dimensionless concentration function
$\beta_{T}:$ Coefficient of thermal expansion
$\beta_{C}:$ Volumetric concentration coefficient
$\theta:$ Dimensionless temperature function
$\eta:$ Dimensionless space variable
$\rho:$ Fluid density
$\sigma:$ Electrical conductivity.

\section{Subscripts}

$\infty$ : Free stream condition

$w$ : Properties at the plate.

\section{Data Availability}

The data available in the manuscript are parameters values only.

\section{Conflicts of Interest}

The authors declare that they have no conflicts of interest.

\section{Acknowledgments}

The authors thank the Nelson Mandela African Institution of Science and Technology, Arusha Technical College, and supervisors for their support.

\section{References}

[1] B. C. Sakiadis, "Boundary-layer behavior on continuous solid surfaces: II. The boundary layer on a continuous flat surface," AIChE Journal, vol. 7, no. 2, pp. 221-225, 1961.

[2] L. J. Crane, "Flow past a stretching plate," Zeitschrift für Angewandte Mathematik und Physik (ZAMP), vol. 21, no. 4, pp. 645-647, 1970.

[3] C. K. Chen and M. I. Char, "Heat transfer of a continuous, stretching surface with suction or blowing," Journal of Mathematical Analysis and Applications, vol. 135, no. 2, pp. 568-580, 1988.

[4] L. E. Erickson, L. T. Fan, and V. G. Fox, "Heat and mass transfer on a moving continuous flat plate with suction or injection," Industrial \& Engineering Chemistry Fundamentals, vol. 5, no. 1, pp. 19-25, 1966.

[5] P. S. Gupta and A. S. Gupta, "Heat and mass transfer on a stretching sheet with suction or blowing," The Canadian Journal of Chemical Engineering, vol. 55, no. 6, pp. 744-746, 1977.

[6] S. Hussain, S. Ahmad, K. Mehmood, and M. Sagheer, "Effects of inclination angle on mixed convective nanofluid flow in a double lid-driven cavity with discrete heat sources," International Journal of Heat and Mass Transfer, vol. 106, pp. 847-860, 2017.

[7] S. Hussain, S. E. Ahmed, and F. Saleem, "Impact of periodic magnetic field on entropy generation and mixed convection," Journal of Thermophysics and Heat Transfer, vol. 32, no. 4, pp. 999-1012, 2018.

[8] O. D. Makinde and W. N. Mutuku, "Hydromagnetic thermal boundary layer of nanofluids over a convectively heated flat plate with viscous dissipation and ohmic heating," UPB Scientific Bulletin, Series A: Applied Mathematics and Physics, vol. 76, no. 2, pp. 181-192, 2014.

[9] S. Hussain, K. Mehmood, and M. Sagheer, "MHD mixed convection and entropy generation of water-alumina nanofluid flow in a double lid driven cavity with discrete heating," Journal of Magnetism and Magnetic Materials, vol. 419, pp. 140-155, 2016.

[10] S. Hussain, "Finite element solution for MHD flow of nanofluids with heat and mass transfer through a porous media with thermal radiation, viscous dissipation and chemical reaction effects," Advances in Applied Mathematics and Mechanics, vol. 9, no. 4, pp. 904-923, 2017.

[11] S. F. Ahmmed, R. Biswas, and M. Afikuzzaman, "Unsteady magnetohydrodynamic free convection flow of nanofluid through an exponentially accelerated inclined plate embedded in a 
porous medium with variable thermal conductivity in the presence of radiation," Journal of Nanofluids, vol. 7, no. 5, pp. 891-901, 2018.

[12] E. Haile and B. Shankar, "Heat and mass transfer through a porous media of mhd flow of nanofluids with thermal radiation, viscous dissipation and chemical reaction effects," American Chemical Science Journal, vol. 4, pp. 828-846, 2014.

[13] M. Uddin, O. A. Bég, A. Aziz, and A. Ismail, "Group analysis of free convection flow of a magnetic nanofluid with chemical reaction," Mathematical Problems in Engineering, vol. 2015, Article ID 621503, 11 pages, 2015.

[14] K. Mehmood, S. Hussain, and M. Sagheer, "Numerical simulation of MHD mixed convection in alumina-water nanofluid filled square porous cavity using KKL model: effects of nonlinear thermal radiation and inclined magnetic field," Journal of Molecular Liquids, vol. 238, pp. 485-498, 2017.

[15] S. Hussain, H. F. Öztop, K. Mehmood, and N. Abu-Hamdeh, "Effects of inclined magnetic field on mixed convection in a nanofluid filled double lid-driven cavity with volumetric heat generation or absorption using finite element method," Chinese Journal of Physics, vol. 56, no. 2, pp. 484-501, 2018.

[16] M. Sheikholeslami, M. T. Mustafa, and D. D. Ganji, "Effect of Lorentz forces on forced-convection nanofluid flow over a stretched surface," Particuology, vol. 26, pp. 108-113, 2016.

[17] M. James, E. Mureithi, and D. Kuznetsov, "Natural convection flow past an impermeable vertical plate embedded in nanofluid saturated porous medium with temperature dependent viscosity," Asian Journal of Mathematics and Applications, vol. 2014, 2014.

[18] M. James, E. W. Mureithi, and D. Kuznetsov, "Effects of variable viscosity of nanofluid flow over a permeable wedge embedded in saturated porous medium with chemical reaction and thermal radiation," International Journal of Advances in Applied Mathematics and Mechanics, vol. 2, no. 3, pp. 101-118, 2015.

[19] P. Sudarsana Reddy and A. J. Chamkha, "Influence of size, shape, type of nanoparticles, type and temperature of the base fluid on natural convection MHD of na nofluids," Alexandria Engineering Journal, vol. 55, no. 1, pp. 331-341, 2016.

[20] M. F. Dimian and M. K. Hadhoda, "Natural convection flows with variable viscosity, heat and mass diffusion along a vertical plate," Mechanics and Mechanical Engineering, vol. 7, no. 2, pp. 61-76, 2004.

[21] D. Hunegnaw and N. Kishan, "Unsteady mhd heat and mass transfer flow over stretching sheet in porous medium with variable properties considering viscous dissipation and chemical reaction," American Chemical Science Journal, vol. 4, no. 6, pp. 901-917, 2014.

[22] N. Shukla and P. Rana, "Unsteady mhd nanofluid flow past a stretching sheet with stefan blowing effect: Ham solution," in Proceedings of the AIP Conference, vol. 1897, 020037 pages, AIP Publishing, 2017.

[23] C. Sulochana and M. K. Kumar, "Numerical investigation of heat and mass transfer in radiative magnetohydrodynamic flow with chemical reaction," International Journal of Advanced Science and Technology, vol. 97, pp. 25-36, 2016.

[24] G. Venkataramanaiah, M. Sreedhar, and M. Lavanya, "Heat generation/absorption effects on magneto-williamson nanofluidflow with heat and mass fluxes," IJEDR (ISSN - 2321-9939), vol. 4, pp. 384-398, 2016.
[25] N. Rao, L. Gahane, and S. Ranganayakulu, "Synthesis, applications and challenges of nanofluids-review," IOSR Journal of Applied Physics, pp. 21-28, 2014.

[26] W. N. Mutuku, "Ethylene glycol (EG)-based nanofluids as a coolant for automotive radiator," Asia Pacific Journal on Computational Engineering, vol. 3, no. 1, article 1, 2016.

[27] A. Alsabery, M. Sheremet, A. Chamkha, and I. Hashim, "Conjugate natural convection of al2o3-water nanofluid in a square cavity with a concentric solid insert using buongiornos twophase model," International Journal of Mechanical Sciences, vol. 136, pp. 200-219, 2018.

[28] P. E. Gharagozloo and K. E. Goodson, "Temperature-dependent aggregation and diffusion in nanofluids," International Journal of Heat and Mass Transfer, vol. 54, no. 4, pp. 797-806, 2011.

[29] S. Mukhopadhyay, "Slip effects on MHD boundary layer flow over an exponentially stretching sheet with suction/blowing and thermal radiation," Ain Shams Engineering Journal, vol. 4, no. 3, pp. 485-491, 2013.

[30] K. Vajravelu, K. Prasad, and C.-O. Ng, "Unsteady convective boundary layer flow of a viscous fluid at a vertical surface with variable fluid properties," Nonlinear Analysis: Real World Applications, vol. 14, no. 1, pp. 455-464, 2013.

[31] A. Ishak, R. Nazar, and I. Pop, "Heat transfer over an unsteady stretching permeable surface with prescribed wall temperature," Nonlinear Analysis: Real World Applications, vol. 10, no. 5, pp. 2909-2913, 2009.

[32] N. Sandeep, C. Sulochana, C. Raju, M. J. Babu, and V. A. Sugunamma, "Unsteady boundary layer flow of thermophoretic mhd nanofluid past a stretching sheet with space and time dependent internal heat source/sink," Applied Mathematics, vol. 10, no. 1, pp. 312-327, 2015.

[33] B. Shanker, B. P. Reddy, and J. A. Rao, "Radiation and mass transfer effects on unsteady mhd free convective fluid flow embedded in a porous medium with heat generation/absorption," Indian Journal of Pure and Applied Physics, vol. 48, pp. 157-65, 2010. 


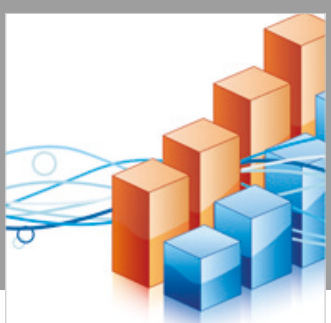

Advances in

Operations Research

\section{-n-m}
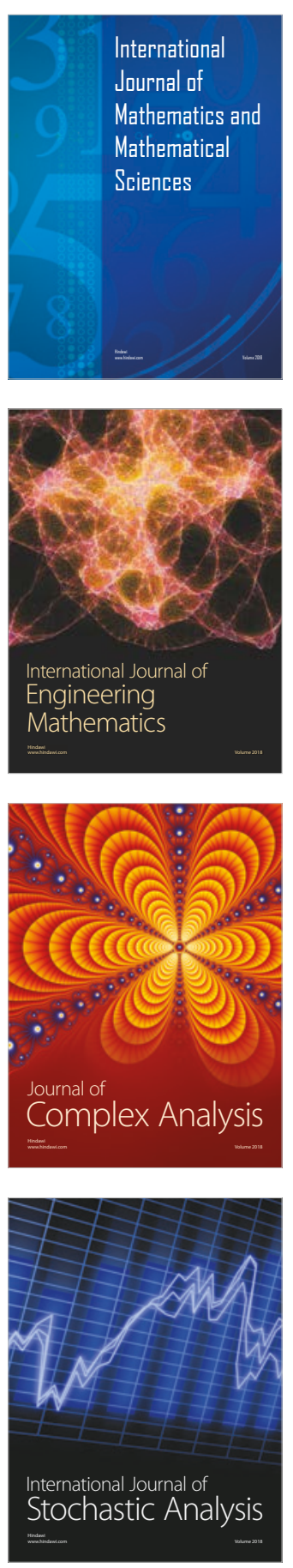
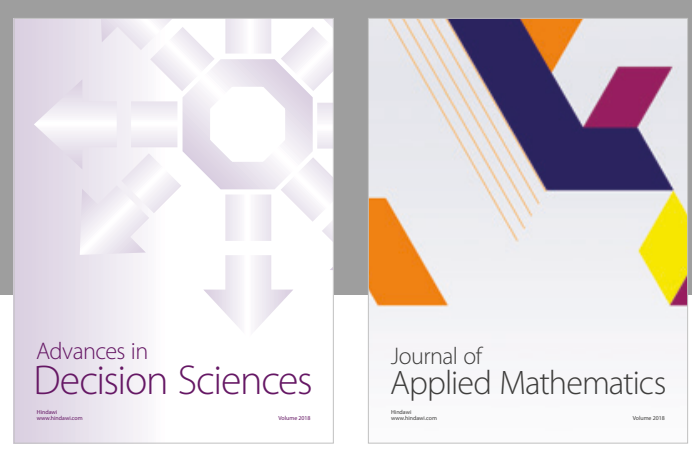

Journal of

Applied Mathematics
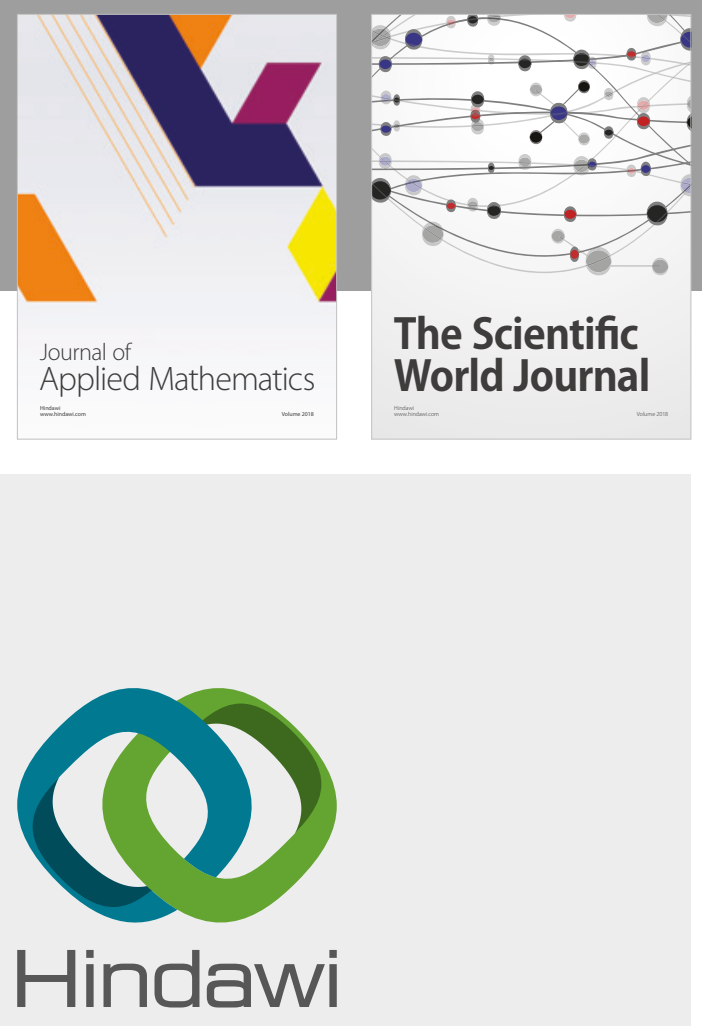

Submit your manuscripts at

www.hindawi.com

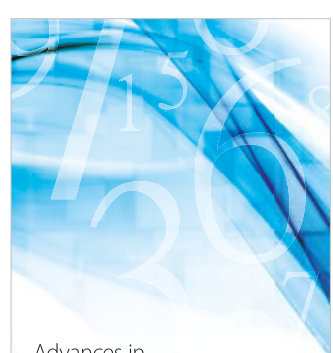

Advances in
Numerical Analysis
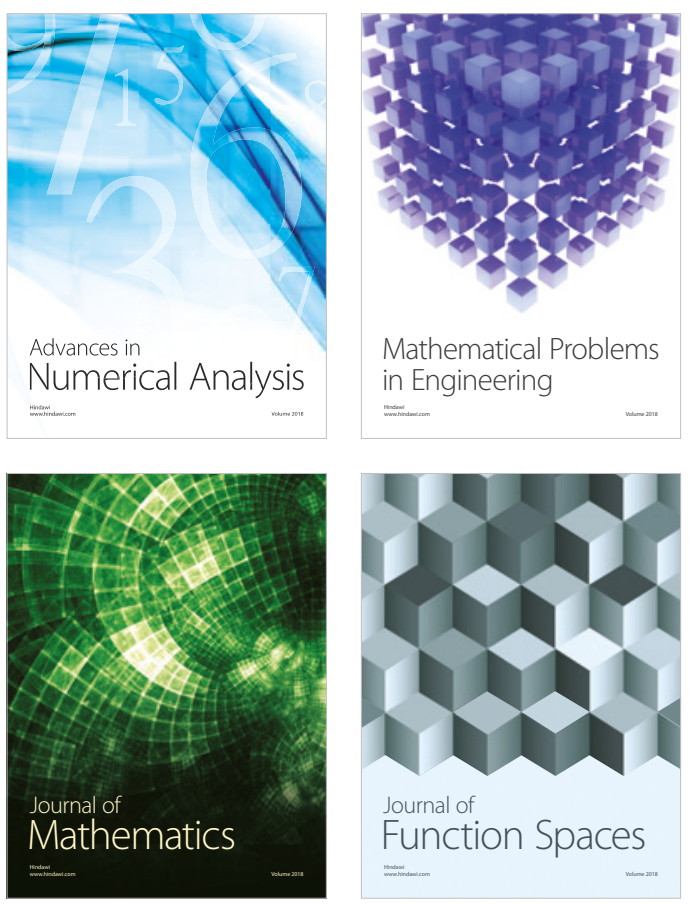

Mathematical Problems in Engineering

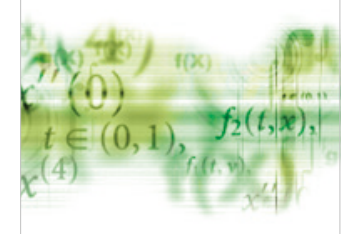

International Journal of

Differential Equations

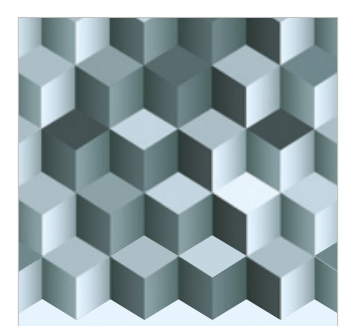

Journal of

Function Spaces

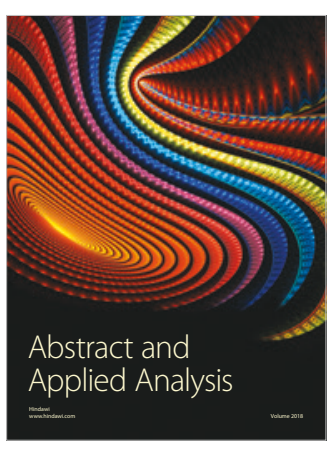

The Scientific

World Journal

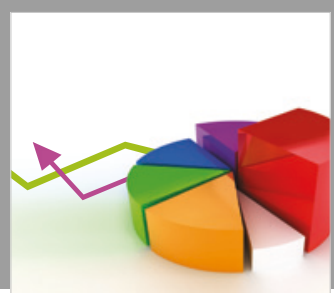

Journal of

Probability and Statistics
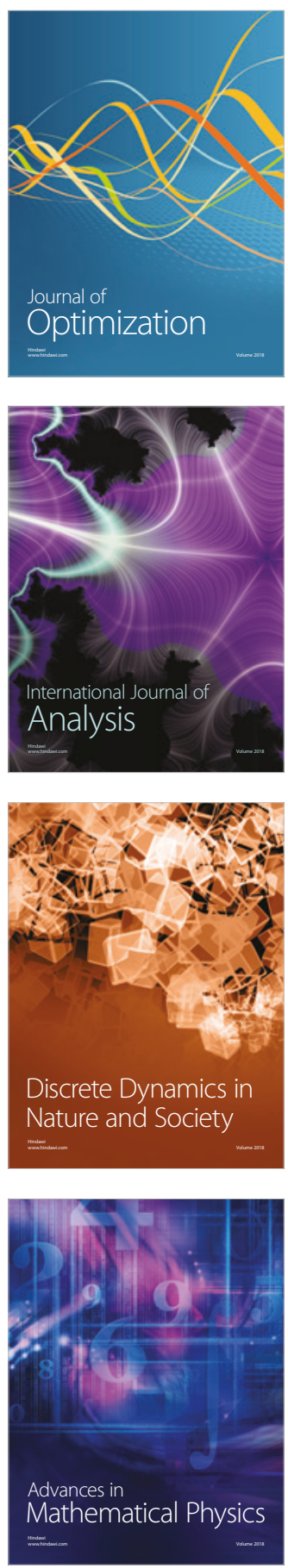